\title{
Capacité de résistance \\ à la sécheresse de la limnée (Limnaea natalensis), Mollusque hôte intermédiaire de Fasciola gigantica, au Sénégal
}

\author{
par G. VASSILIADES $(*)$
}

\begin{abstract}
RÉSUMÉ
Une étude expérımentale faite au Sénégal sur une souche de Limnaea natalensis démontre la capacité de résistance de cette espèce à une période de sécheresse de l'ordre de 15 à 30 jours chez les grosses limnées adultes et de 60 à 90 jours chez les petites limnées.

Cette aptitude à résister doit être prise en considération pour fixer le moment propice à l'épandage de molluscicides. La période correspondant au début de l'hivernage est proposée.
\end{abstract}

\section{INTRODUCTION}

Limnaea natalensis, Mollusque Gastéropode de l'ordre Basommatophora, est l'hôte intermédiaire de Fasciola gigantica, agent causal de la distomatose, maladie considérée comme l'une des affections parasitaires les plus graves frappant les Ruminants domestiques d'Afrique.

Les travaux de divers auteurs dont GRETILLAT $(1961 a, b)(3,4)$ au Sénégal et DAYNES (1967) (2) à Madagascar, ont permis de bien connaître le cycle évolutif de $F$. gigantica et montré le rôle d'hôte intermédiaire joué par L. natalensis.

La connaissance de la biologie de ce Mollusque est donc fondamentale pour l'élaboration de tout programme de lutte contre la distomatose par destruction de son hôte intermédiaire.

Il est généralement admis que $L$. natalensis est un Mollusque aquatique strict, vivant tou-

(*) I. S. R. A., Laboratoire national de l'Elevage et de Recherches vétérinaires, B. P. 2057, Dakar, Sénégal. jours immergé dans l'eau douce et incapable de survivre plus de quelques jours en absence complète d'eau. Toutefois, les résultats indiscutables obtenus par BITAKARAMIRE au Kenya (1968) (1) et des expériences effectuées à Madagascar en 1972 (6) démontrent que $L$. natalensis est capable de résister un certain temps sans eau. BITAKARAMIRE a démontré en effet que L. natalensis peut survivre pendant au moins 56 à 168 jours en période de sécheresse, tandis qu'à Madagascar il est prouvé que $L$. natalensis hovarum est capable de survivre sans « eau libre " au moins pendant 22 à 27 jours.

Cette aptitude peut avoir d'importantes conséquences d'ordre épidémiologique. De plus, sur le plan pratique, elle est de nature à modifier les données généralement admises pour la conduite des opérations de destruction de ces limnées par épandage de molluscicides.

Aussi, au Sénégal, où la majeure partie du pays est soumise à une saison sèche très marquée, il est apparu nécessaire de reprendre ces expériences pour voir dans quelle mesure la souche sénégalaise de $L$. natalensis est capable de résister à une longue période de sécheresse. 
Dans ce but, une étude expérimentale a été réalisée sur un élevage de $L$. natalensis soumis à des périodes d'assèchement de plus en plus longues. Les résultats de ces expériences sont présentés dans cette note.

\section{MATÉRIEL ET MÉTHODES}

Les limnées sont récoltées dans un marigot de Sangalkam, à $37 \mathrm{~km}$ à l'est de Dakar et ramenées au laboratoire où elles sont mises en élevage dans 1 des 10 bacs en ciment situés dans un bâtiment spécialement conçu pour l'élevage des Mollusques (GRETILLAT, 1964) (5).

Chaque bac utilisé, long de $215 \mathrm{~cm}$ et large de $79 \mathrm{~cm}$, est rempli d'eau du robinet jusqu'à une hauteur de $18,5 \mathrm{~cm}$, soit un volume d'eau égal à 3141 . Le fond est constitué de sable fin, d'une épaisseur de $8 \mathrm{~cm}$. Une végétation aquatique se constitue rapidement après repiquage de plants d'Ericaulon et le développement d'algues vertes.

L'alimentation des limnées est assurée par des feuilles de laitue fraîche.

L'assèchement du bac se fait par vidange grâce à un dispositif d'évacuation prévu à cet effet au niveau de la surface du fond de sable. Au bout d'1 h, le bac est complètement vidé. Le sable s'assèche alors progressivement de la surface vers le fond, en 1 semaine, par évaporation. A ce stade, la surface se couvre d'une pellicule, formée de débris organiques, d'algues vertes et de sable, qui se craquèle rapidement.

Pour le contrôle de viabilité des Mollusques en cours d'expérience, 20 d'entre eux sont récoltés au hasard dans le bac et mis avec de l'eau dans une boîte de pétri. Dans les minutes qui suivent, chez les limnées vivantes, le corps rétracté et immobile au fond de sa coquille se met en mouvement (observation à la loupe binoculaire à faible grossissement). Le décompte définitif a lieu $1 \mathrm{~h}$ après immersion. Pour l'ensemble du bac, les Mollusques sont observés directement dans leur milieu, $24 \mathrm{~h}$ après la remise en eau.

\section{RÉSULTATS}

Expérience $1\left(\mathrm{bac} \mathrm{n}^{\circ} 1\right)$

- Situation avant l'assèchement: (29.11.75) souche élevée normalement depuis 1 mois comprenant de grosses limnées (taille moyenne : $14,5 \mathrm{~mm}$ ) et des limnées filles âgées de moins d'1 mois.

- Durée de l'assèchement : 16 jours.

- Observations $24 h$ après la remise en eau: toutes les grosses limnées sont mortes. Par contre les jeunes limnées sont toutes vivantes.

Dès ce $1^{\text {er }}$ essai, il est apparu que les plus grosses limnées sont sensibles à l'assèchement puisqu'aucune d'elles n'a survécu après 16 jours sans eau. Par contre, les jeunes limnées, nées quelques jours avant la mise à sec, ont parfaitement résisté. Cette observation se confirmera au cours des essais suivants.

\section{Expérience 2 (même bac)}

- Situation avant l'assèchement : nombreuses limnées filles provenant de l'expérience 1, après 1 mois de croissance normale (16.1.76) et donc âgées de 2 mois. Leur taille moyenne varie entre 6 et $7 \mathrm{~mm}$.

- Durée de l'assèchement : 49 jours.

- Contrôles de viabilité : au $28^{\mathrm{e}} \mathrm{j}$, toutes les limnées contrôlées sont vivantes; puis le pourcentage de mortalité passe de 40 p. 100 au $35^{\mathrm{e}}$ jour, à 70 p. 100 le $42^{\mathrm{e}}$ jour et à 80 p. 100 le $49^{\mathrm{e}}$ jour. La croissance est nulle pendant toute la durée de l'assèchement.

- Croissance des limnées survivantes après la remise en eau : en 45 jours, la taille moyenne des limnées est passée de $6,675 \mathrm{~mm}$ à $10 \mathrm{~mm}$ soit une croissance de $3,325 \mathrm{~mm}$ pour un âge réel de 3 mois et 15 jours (la durée de la période d'assèchement, soit 49 jours, n'étant pas comptée).

$\mathrm{Ce} 2^{\mathrm{e}}$ essai ne portait donc que sur des petites limnées. Après 49 jours d'assèchement, 20 p. 100 de celles-ci sont encore vivantes, ce qui constitue déjà un résultat appréciable. Au cours de cette " diapause ", les limnées se rétractent au maximum au fond de leur coquille, dont l'ouverture se plaque contre le sol et restent fixées à sa' surface, immobiles, sans chercher à s'enfouir (cf. photos). Il n'existe aucun dispositif spécial de fermeture. Pendant toute la période d'assèchement, les limnées vivent sur leurs réserves car il n'y a pas d'alimentation et la croissance est interrompue. Dès la remise en eau et la reprise de l'alimentation, la croissance reprend normalement malgré la longue interruption due à l'assèchement. 


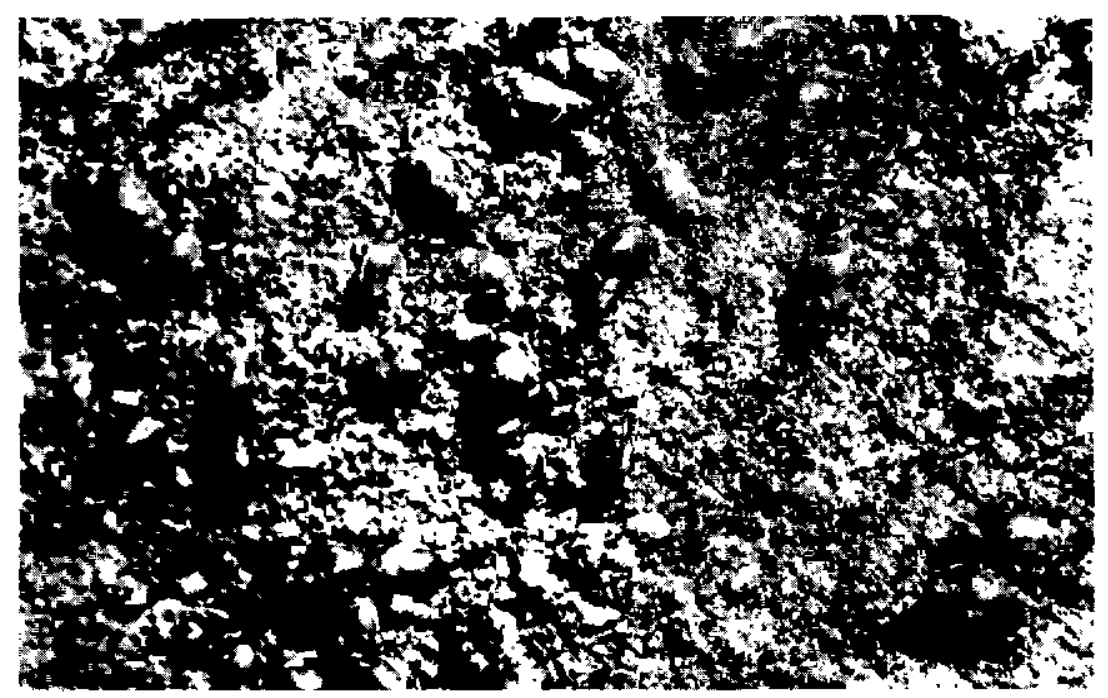

Photo $\mathrm{n}^{0}$ 1. - Groupe de limnées en « diapause » sur fond de sable asséché.

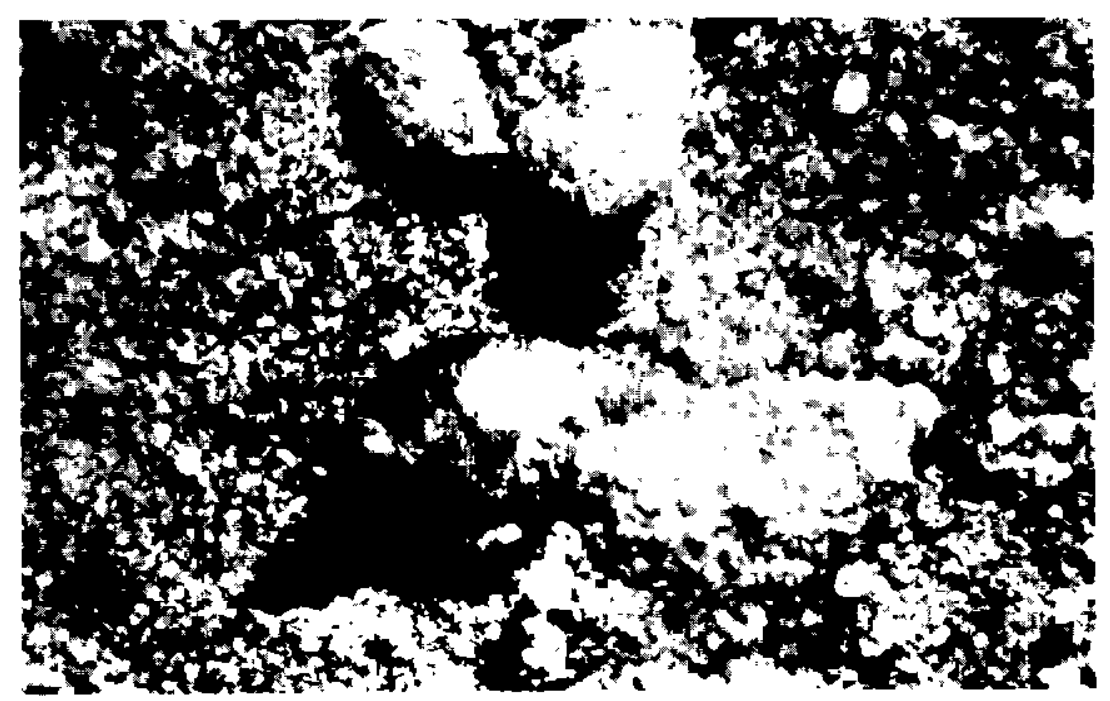

Photo $n^{\circ}$ 2. - Une limnée en « diapause » (gros plan).

Expérience 3 (même bac)

- Situation avant l'assèchement : (23.4.76) nombreuses limnées filles provenant de l'expérience précédente, âgées de 3 à 4 moss et mesurant en moyenne $10 \mathrm{~mm}$.

- Durée de l'assèchement : 60 jours.

- Contrôles de viabilité : au $30^{\mathbf{e}}$ jour, la mortalité est de 70 p. 100 , au $45^{\mathrm{e}}$ jour : 75 p. 100 . Au $60^{\mathrm{e}}$ jour, la mortalıté est très élevée, proche de 100 p. 100 . Le bac est alors remis en eau le 19.6.76 pour sauver la souche.
Dans cet essai, la mortalité a été très importante mais les quelques limnées rescapées ont quand même résisté 2 mois et suffisamment de limnées ont survécu pour qu'après la remise en eau du bac la souche soit maintenue.

Expérience 4 (même bac)

A la fin de l'expérience précédente, les limnées survivantes ont repris leur croissance et se sont multipliées normalement. Des naissances sont observées le 12.8.76 à partir de pontes émises le 23.7.76, soit après 20 jours d'incubation. 
Un mo1s après, les 2 générations sont séparées. Les jeunes limnées âgées d'1 mois restent dans le même bac, les limnées mères sont mises dans un autre bac $\left(\mathrm{n}^{\circ} 2\right)$.

\section{- Croissance des limnées:}

Bac 1 (limnées filles) : en 25 jours, du 18.9.76 au 12.10.76, la taille moyenne des limnées est passée de $9 \mathrm{~mm}$ (âge 38 jours) à $12,90 \mathrm{~mm}$ (62 jours), soit une croissance de $3,9 \mathrm{~mm}$.

Bac 2 (limnées mères) : pendant la même période, la taille moyenne est passée de $12,55 \mathrm{~mm}$ à $15,60 \mathrm{~mm}$, soit une croissance de $3,05 \mathrm{~mm}$. A ce stade, une nouvelle série de pontes est observée.

\section{- Situation avant l'assèchement :}

Bac 1 : limnées âgées de 2 mois $(12,90 \mathrm{~mm})$.

Bac 2 : limnées mères $(15,60 \mathrm{~mm})$ et nouvelle génération issue de pontes du 12.10.76.

- Durée de l'assèchement : 25 jours.

- Contrôles de viabilité :

bac 1

- au $10^{\circ}$ jour : 5 p. 10050 p. 100 (contrôles unique-

- au $15^{\circ}$ jour : 50 p. $100 \quad 55$ p. 100

- au $25^{\mathrm{e}}$ jour : 95 p. $100 \quad 95$ p. 100

La période d'assèchement a été écourtée en raison de la très forte mortalité enregistrée. Les bacs sont remis en eau pour assurer la survie de la souche qui n'est cependant plus utilisée dans les expériences suivantes.

Dans cette expérience, 2 groupes d'âges ont été testés : des limnées de 2 mois et des limnées plus âgées. Une très forte mortalité est notée dans les 2 groupes. Cependant 10 jours après l'assèchement, on note une différence importante dans les 2 lots puisque la mortalité n'est que de 5 p. 100 chez les petites limnées, contre 50 p. 100 chez les autres. En fait, comme on va le voir dans les essais suivants, les limnées des 2 groupes avaient atteint au départ une trop grande taille, incompatible avec une bonne aptitude de résistance à la sécheresse.

\section{Expérience 5 (bac $\mathrm{n}^{0} 3$ )}

(Matériel nouveau, de même origine).

- Situation avant l'assèchement: (4.1.77) limnées de taille moyenne égale à $17,8 \mathrm{~mm}$ et nombreuses petites limnées âgées de 15 jours.

- Durée de l'assèchement : 35 jours.
- Contrôles de viabilité :

- Au 11e jour : 75 p. 100 de mortalité chez les grosses limnées ; pas de mortalité chez les petites.

- Au $35^{\mathrm{e}}$ jour : toutes les grosses limnées sont mortes; toujours pas de mortalité chez les plus petites. Le bac est remis en eau et les jeunes limnées reprennent alors normalement leur croissance. L'expérience est poursuivie dans un autre bac sur une plus longue période.

Ce test n'a donc porté que sur des grosses limnées. Dès le $11^{\mathrm{e}}$ jour d'assèchement, 75 p. 100 d'entre elles sont déjà mortes et à 35 jours, il n'y a plus aucune limnée encore vivante. Par contre, à ce stade, les petites limnées qui se trouvaient dans le bac au moment de la mise à sec sont encore toutes vivantes.

\section{Expérience 6 (bac n 4)}

- Situation avant l'assèchement (4.1.77) limnées de taille moyenne égale à $19,6 \mathrm{~mm}$ et nombreuses petites limnées âgées de 15 jours.

- Durée de l'assèchement: 98 jours.

- Au 11e jour: pas de mortalité chez les petites limnées, mais 70 p. 100 de mortalité chez les plus grosses.

- Au $35^{\mathrm{e}}$ jour : toutes les grosses limnées sont mortes, comme dans l'expérience précédente (taille moyenne supérieure à $10 \mathrm{~mm}$ ). Chez les petites limnées (taille moyenne : $6,2 \mathrm{~mm}$ ) la mortalité est de 10 p. 100.

Durée p. 100

de l'assèchement de mortalité

-11 jours $\ldots \ldots \ldots \ldots \ldots \ldots \ldots . \ldots$

-35 jours $\ldots \ldots \ldots \ldots \ldots \ldots \ldots .10$

-42 jours $\ldots \ldots \ldots \ldots \ldots \ldots \ldots . \ldots 10$

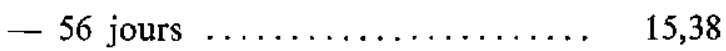

- 63 jours ............... 50

-70 jours $\ldots \ldots \ldots \ldots \ldots \ldots \ldots \ldots .62,5$

- 77 jours $\ldots \ldots \ldots \ldots \ldots \ldots \ldots \ldots, 66,66$

-84 jours $\ldots \ldots \ldots \ldots \ldots \ldots \ldots \ldots \quad 73,3$

-91 jours $\ldots \ldots \ldots \ldots \ldots \ldots \ldots .65$

- 98 jours $\ldots \ldots \ldots \ldots \ldots \ldots \ldots .100$

$\mathrm{Au}$ cours de cet essai, le degré hygrométrique a varié de 86 à 95 p. 100 le matin à 6 h et de 44 à 67 p. 100 à 15 h. 
Jusqu'au $35^{\mathrm{e}}$ jour donc, les résultats sont identiques à l'essai précédent et l'expérience est poursuivie avec les petites limnées. Celles-ci ont survécu à 91 jours d'assèchement avec un pourcentage de mortalité de 85 p. 100 . A 98 jours, la mortalité a été totale.

\section{CONCLUSIONS}

Les résultats obtenus au cours des diverses expériences réalisées permettent de conclure qu'il existe bien une capacité de résistance à la sécheresse de Limnaea natalensis au Sénégal.

Cette aptitude est en rapport avec la taille des limnées et par conséquent avec leur âge. Dans les conditions de notre expérimentation, les grosses limnées, de taille moyenne supérieure à $10 \mathrm{~mm}$ et âgées de 3 mois et plus, ont une capacité de résistance limitée mais néanmoins réelle de 15 à 30 jours. Les petites limnées, de taille moyenne inférieure à $10 \mathrm{~mm}$, et âgées de moins d'un mois, sont beaucoup plus résistantes puisqu'elles peuvent survivre après une période d'assèchement de 60 à 90 jours. Dans tous les cas, le pourcentage de mortalité est de plus en plus élevé à mesure que la sécheresse se prolonge, mais le nombre de limnées survivantes est suffisant pour assurer la pérennité de l'espèce après le retour des conditions normales.

Au cours de la "diapause » les limnées s'immobilisent sur la surface du sable sec sans chercher à s'enfouir dans le sol, cessent de se nourrir et de croitre, vivant sur leurs réserves. Dès le retour de l'eau et la reprise de l'alimentation, les limnées encore vivantes reprennent un développement normal sans être apparemment affectées par cette interruption.

La diffẻrence observée entre les grosses limnées et les petites limnées quant à leurs capacités respectives de résistance, peut s'expliquer par une plus grande exigence en eau chez les limnées adultes de grande taille, tandis que les petites limnées doivent pouvoir utiliser l'humi- dité de l'air pour satisfaire en partie leurs faibles besoins en eau en rapport avec leur petit format (jusqu'à 95 p. 100 d'humidité dans l'expérience 6).

La capacité de résistance augmente donc au fur et à mesure que décroît la taille des limnées et inversement.

On peut penser que dans la nature, au niveau des mares, les choses se passent de la même façon. $\mathrm{Au}$ moment de l'assèchement des mares, en saison sèche, les grosses limnées meurent assez rapidement mais les plus petites, nées quelques semaines seulement avant l'assèchement critique, peuvent survivre 2 à 3 mois selon les conditions climatiques et en particulier le degré hygrométrique, et attendre ainsi le retour de l'eau, aux premières pluies, pour recoloniser la mare temporairement asséchée.

Dans ces conditions, il est nécessaire de reconsidérer l'opinion antérieurement admise selon laquelle il ne peut exister de distomatose dans les zones où les mares s'assèchent périodiquement à un moment de l'année, surtout quand cet assèchement n'excède pas 2 à 3 mois. Cette notion doit être désormais prise en considération dans les enquêtes épidémiologiques et les prospections malacologiques. Sur le plan pratique, le moment généralement recommandé pour l'épandage de molluscicides est la période des plus basses eaux pour toucher le maximum de limnées concentrées dans les derniers bas-fonds avec une quantité minimale de produit, toujours coûteux. Dans ce cas, les limnées en « diapause » dans les zones asséchées, seraient épargnées et pourraient recoloniser le gîte traité. Aussi, à ce sujet, nous partageons entièrement l'opinion de BITAKARAMIRE (1968) (1) qui propose de choisir plutôt la période correspondant à quelques semaines après les premières pluies, donc en début d'hivernage, quand toutes les limnées sont alors immergées dans l'eau. Cette solution, bien que plus coûteuse, aurait plus de chance de donner de bons résultats.

\section{SUMMARY}

Snail resistance to dryness. The case of Limnaea natalensis,
an intermediate host of Fasciola gigantica in Senegal

In an experimental study carried out in Senegal on a strain of Limnaea natalensis, it was shown that this species could survive in drought situations for periods of 15 to 30 days in case of large sized adults and for 60 to 90 days in case of young snails of small size.

As a result, it is suggested that the optimal time for the application of molluscicides should be the begining of the rainy season. 


\section{RESUMEN}

Capacidad de resistencia a la sequedad de Limnaea natalensis, molusco huesped intermediario de Fasciola gigantica en Senegal

Un estudio experimental efectuado en Senegal sobre una cepa de Limnaea natalensis demuestra la capacidad de resistencia de dicha especie a un periodo de sequedad de unos 15 a 30 días en las gruesas limneas adultas y de unos 60 a 90 dias en las pequeñas limneas.

Se necesita tener en cuenta esta resistencia para elegir el momento prop1cio para el esparcımiento de helicidas. Se propone el periodo corespondiendo al principio de la invernada.

\section{BIBLIOGRAPHIE}

1. BITAKARAMIRE (P. K.). The survival of Lymnaea natalensis in drought conditions. Bull. epizoot. Dis. Afr., 1968, $16: 473-475$.

2. DAYNES (P.). La distomatose à Madagascar. Cycle de Fasciola gigantica. Rev. Elev. Méd. vét. Pays trop., $1967,20(4): 557-562$.

3. GRETILLAT (S). Note préliminaire sur l'épidémiologie de la distomatose bovine au Sénégal. Rev. Elev. Méd. vét. Pays trop., 1961, 14 (3) : 283-291.

4. GRETILLAT (S.). Distomatose et bilharziose des ruminants domestiques. Leur prophylaxie par la lutte anti-mollusques. Rev. Elev. Méd. vét. Pays trop., 1961, 14 (3) : 293-313.

5. GRETILLAT (S.). Rapport sur des travaux réalisés depuis le 1er janvier 1964 à l'aide d'un crédit de 5000 dollars fournis par l'Organisation mondiale de la Santé à Genève. Dakar, L. N. E. R. V., 1964, $17 \mathrm{p}$.

6. Rapport annuel 1972. Région de recherches de Madagascar. I. E. M. V. T. Résistance de la limnée à l'assèchement des gîtes, p. 79-84. 